\title{
Pre-implantation developmental potential from in vivo and in vitro matured mouse oocytes: a cytoskeletal perspective on oocyte quality
}

\author{
Alexandra Sanfins • Carlos E. Plancha • David F. Albertini
}

Received: 21 July 2014 / Accepted: 7 October 2014 / Published online: 9 November 2014

(C) The Author(s) 2014. This article is published with open access at Springerlink.com

\begin{abstract}
Purpose In the present study, fertilization and developmental potential of mouse oocytes matured in different conditions were tested. The efficiency of in vitro fertilization (IVF), preimplantation development and some important aspects of cytokinesis during early cleavages are discussed.

Methods In vivo matured (IVO), in vitro matured (IVM) and roscovitine-treated (IVM-Rosco) mouse oocytes were subjected to IVF under identical conditions. Three replicates per group were analyzed. Fertilization was identified by the presence of two pronuclei at $6-8 \mathrm{~h}$ post-fertilization. Evaluation of pre-implantation embryonic development was done daily from day 2 to day 5 and embryos were processed for analyses of chromatin, nuclear lamina, microtubules and centrosomal proteins by conventional and confocal fluorescence microscopy.
\end{abstract}

Capsule Comparisons of cytoskeletal reorganization in mouse embryos derived from in vivo or in vitro matured oocytes suggest that morphokinetic manisfestations of embryo quality reside in spatial and temporal differences established during the final stages of oocyte maturation.

A. Sanfins

Faculdade de Medicina Veterinária, Universidade Lusófona de Humanidades e Tecnologias, Campo Grande 376, 1749-024 Lisbon, Portugal

C. E. Plancha $(\bowtie)$

Unidade de Biologia da Reprodução, Instituto de Histologia e

Biologia do Desenvolvimento, Faculdade de Medicina da

Universidade de Lisboa, 1649-028 Lisbon, Portugal

e-mail: cplancha@fm.ul.pt

C. E. Plancha

Centro Médico de Assistência à Reprodução - CEMEARE, Rua

Alfredo Mesquita, 2E, 1600-922 Lisbon, Portugal

D. F. Albertini

Department of Molecular and Integrative Physiology, University of Kansas Medical Center, 3901 Rainbow Boulevard, Kansas City, KS 66160-7401, USA
Results Both IVM groups displayed lower fertilization rates when compared to in vivo controls. While IVO-derived embryos exhibit efficient and synchronous progression to the blastocyst stage, both IVM-derived embryos exhibit a delay in embryonic progression, and a lower blastocyst rate. Interestingly, IVM-Rosco M-II oocytes exhibited more blastomere symmetries and higher number of cells at the blastocyst stage than the IVM group with the most notable influence being on the centrosome-microtubule complex of blastomeres.

Conclusion Our study strongly indicates that when compared to spontaneously in vitro matured oocytes, treatment with roscovitine may partially enhance developmental competence by maintaining coordination between nuclear and cytoplasmic events. Further evidence is given of cytoskeletal biomarkers that can be identified during in vitro oocyte maturation conditions.

Keywords Oocyte maturation · Blastocyst · Cell cycle arrest . Roscovitine $\cdot$ Blastomere

\section{Introduction}

Oocyte maturation is the final phase of oogenesis during which a number of complex changes take place that involve progression of the oocyte from prophase-I to metaphase-II [1, 2]. This process involves coordination of nuclear and cytoplasmic events necessary to produce quality oocytes capable of being fertilized and support preimplantation development [3]. In vivo, this process depends on the close interaction between the cumulus cells and the oocyte involving a cascade of signaling pathways, particularly EGF-like proteins produced from the cumulus cells in response to LH [4]. Since the original experiments of Pincus \& Enzman (1935) and Edwards (1965) it became clear that oocytes undergo immediate and spontaneous maturation once removed from their 
follicular environment $[5,6]$. However, while IVM supports nuclear maturation, cytoplasmic maturation, a process required for the expression of developmental competence, is not assured [3]. Overcoming the obstacles limiting cytoplasmic maturation during IVM remains a challenge for the production of quality oocytes of clinical value to the practice of human assisted reproductive technologies (ARTs) [7]. Several features have been described that might contribute to the high developmental competence of IVO oocytes when compared to their IVM counterparts, such as ATP concentration [8], number and distribution of active mitochondria [9], glutathione content [10], transcriptional and translational activity $[11,12]$, and imprinting/ methylation patterns in embryos derived from IVO and IVM [13]. Our group has been especially interested in analyzing patterns of cytoskeletal organization in IVO and IVM matured oocytes to better define properties indicative of developmental competence. Specifically, when spindle size / shape and distribution of cytoplasmic Microtubule Organizing Centers (MTOCs) were analyzed, IVO oocytes exhibited compact and pointed-shaped spindles with increased number of cytoplasmic MTOCs. In contrast, IVM oocytes displayed barrel-shaped spindles, which exhibited much more $\gamma$-tubulin aggregates at the spindle poles, associated with a low number of cytoplasmic MTOCs [14]. These features were found to result from the distinct cell cycle regulation with differential allocation of $\gamma$-tubulin stores during the first $5 \mathrm{~h}$ of oocyte maturation [15]. Thus, spindle morphogenesis in IVM oocytes favors recruitment of $\gamma$-tubulin to the spindle, while retention of $\gamma$-tubulin foci in the cytoplasm with reduced $\gamma$-tubulin recruitment to the spindle is observed in IVO oocytes. Consequently, depletion of maternal factors, like $\gamma$-tubulin, from the cytoplasm is observed upon IVM and expected to increase with emission of the second polar body after fertilization [16-18]. These critical differences in cytoskeleton organization were found to be a result of the distinct cell cycle progression between IVO and IVM oocytes. In fact, in IVO oocytes the follicular environment maintains the G2/M cell cycle delay necessary to keep nuclear lamina integrity and proper localization of cytoplasmic MTOCs which is not observed under IVM conditions [15].

The communication established between the oocyte and its surrounding cumulus cells is crucial to promote the oocyte nuclear and cytoplasmic maturation needed to achieve developmental competence [17-20]. When a G2/M phase delay was imposed in culture by treating oocytes with the specific MPF inhibitor, roscovitine, IVO-like cytoskeletal characteristics were partially achieved in IVM oocytes (IVM-Rosco), namely pointed-shape and cortically localized spindles associated with an increased number of cytoplasmic MTOCs [15] suggesting that an imposed delay in cell cycle progression may be necessary to allow nuclear changes to wait for the cytoplasmic maturation events.
In the present study, additional parameters of oocyte quality, in particular fertilization and developmental competencies are evaluated after careful comparison of the pre-implantation development characteristics between embryo-derived IVO, IVM and IVM-Rosco oocytes. Special emphasis is placed on the expression of cytoskeletal proteins involved in cell polarity determination and stability of the nucleus.

\section{Material and methods}

Animals

All oocytes were obtained from CF-1 outbred mice (HarlanSprague Dawley, Indianapolis, IN) between 6 and 8 week of age. Animals were handled according to the Guide for Care and Use of Laboratory Animals (National Academy of Science, 1996) and maintained on a 14L:10D photoperiod under constant temperature and relative humidity conditions. Food and water were provided ad libitum. Five females were kept per cage and a total of 50 females were used for this study. For IVF and mating experiments sperm was obtained from B6D2F1 hybrid male mice (Harlan-Sprague Dawley, Indianapolis, IN) between 8 and 12 week-old. Sexually matured males of proven fertility were used after successful mating and after abstaining for 1 week.

\section{Collection and culture of oocytes}

For all experiments three types of oocyte collections were preformed with either IVO oocytes or IVM oocytes. IVO oocytes were obtained from mice previously injected $46 \mathrm{~h}$ earlier with $5 \mathrm{IU}$ of equine chorionic gonadotropin (eCG, CalBiochem) to stimulate follicular development followed $46 \mathrm{~h}$ later by $5 \mathrm{IU}$ of human chorionic gonadotropin (hCG, Sigma). Oocytes were collected from the swollen ampulla $15 \mathrm{~h}$ post-hCG in collection medium consisting of Hepesbuffered Eagles MEM with Hanks'salts supplemented with $100 \mathrm{IU} / \mathrm{ml}$ penicillin, $100 \mu \mathrm{g} / \mathrm{ml}$ streptomycin and $0.3 \%$ BSA. Only MII-stage oocytes were isolated.

IVM oocytes were obtained from mice injected $46 \mathrm{~h}$ earlier with $5 \mathrm{IU}$ of eCG and cumulus-enclosed oocytes (COCs) were isolated by follicular puncture in collection medium. COCs were cultured for $15 \mathrm{~h}$ in in vitro maturation medium (IVM oocytes) consisting of Eagle's MEM supplemented with Earle's salts, $2 \mathrm{mM}$ glutamine, $0.23 \mathrm{mM}$ pyruvate, $100 \mathrm{IU} / \mathrm{ml}$ penicillin, $100 \mu \mathrm{g} / \mathrm{ml}$ streptomycin, $0.3 \%$ BSA and $1 \mathrm{mg} / \mathrm{ml}$ fetuin (SIGMA) which had been previously dialyzed against MEM for 2 days using a slide-A-lyzer dialysis cassette (Pierce Biotechnology, Rockford, IL). Only MII-stage oocytes were isolated and used for these experiments. These oocytes will be referred as IVM oocytes. In parallel COCs were cultured in in vitro maturation medium containing $50 \mu \mathrm{M}$ Roscovitine 
(BIOMOL, Pennsylvania, USA) for $3 \mathrm{~h}$. Following roscovitine treatment, oocytes were washed three times in 100x volume of maturation medium without roscovitine and were subsequently allowed to mature in vitro for $15 \mathrm{~h}$ without drug as described above. Only MII-stage oocytes were isolated and used for these experiments. These oocytes will be referred as IVMRosco oocytes. All cultures were preformed in a humidified atmosphere of $5 \% \mathrm{CO}_{2}$ in air at $37^{\circ} \mathrm{C}$.

In vitro fertilization and embryo culture

IVF and embryo culture were preformed as previously described only using M-II oocytes identified in each group [8]. IVO, IVM and IVM-Rosco oocytes were fertilized and embryos cultured under the same conditions. Additionally, pronuclear (PN) stage embryos were isolated upon visualization of vaginal plugs in females that received hCG and were mated with males of proven fertility. A total of 3 full experiments were performed. All culture media was prepared fresh using frozen reagents (Sigma Biosciences) kept at $-20{ }^{\circ} \mathrm{C}$ for up to 2 months. Drops of media overlaid with filtered embryo-tested mineral oil (Sigma Biosciences) were set up the day before and allowed to equilibrate overnight in a humidified atmosphere of $5 \% \mathrm{CO}_{2}$ in air at $37{ }^{\circ} \mathrm{C}$. Media composition is described elsewhere [21, 22].

Sperm was collected from caudae epididymes and placed in a $200 \mu \mathrm{l}$ drop of modified Tyrode medium under oil in a $35 \mathrm{~mm}$ Falcon dish. Epididymal content was released using watchmaker's forceps and allowed to swim out for $10 \mathrm{~min}$. Subsequently, epididymes were removed and a $100 \mu$ volume of this preparation was diluted 1:4 in modified Tyrode medium under oil for capacitation in 4 well-Nunc dishes. Sperm was allowed to capacitate for $90 \mathrm{~min}$ at $37^{\circ} \mathrm{C}$ in a humidified atmosphere of $5 \% \mathrm{CO}_{2}$ in air. During this period sperm concentration was determined using a hemocytometer.

IVF was preformed using only M-II oocytes produced from the following groups: 1) M-II IVO oocytes collected from the ampulla at $15 \mathrm{~h}$ post-hCG; 2) M-II IVM and 3) M-II IVM-Rosco oocytes previously cultured for $15 \mathrm{~h}$ as described above. For collection of IVO oocytes oviducts were placed into Hepes-free PBS supplemented with $100 \mathrm{IU} / \mathrm{ml}$ penicillin and $100 \mu \mathrm{g} / \mathrm{ml}$ streptomycin as described [8]. A single oviduct was placed directly into the oil surrounding the 200- $\mu$ l drop of modified potassium simplex optimization media (KSOM) [22]. Intact cumulus masses were released from the ampulla and gently placed into the drop of modified KSOM. This procedure allows the transfer of cumulus masses with the minimum amount of debris and PBS into the fertilization drop. Following collection of the cumulus masses, capacitated sperm was added to the fertilization drop at a final motile concentration of 800,000-900,000 sperm/ml. For fertilization of both IVM and IVM-Rosco oocytes, cumulus cells were removed by gentle pipetting and oocytes were extensively washed in $50 \mu \mathrm{l}$ drops of modified KSOM media. Subsequently, oocytes were transfer with minimum amount of media into the $200 \mu \mathrm{l}$ drop of modified KSOM and sperm added as above. Fertilization was carried out for $4 \mathrm{~h}$ at $37^{\circ} \mathrm{C}$ in a humidified atmosphere of $5 \% \mathrm{CO}_{2}$ in air. $4 \mathrm{~h}$ later the eggs were washed several times in $50 \mu$ drops of KSOM [21]. 10 15 zygotes were placed in $50 \mu \mathrm{l}$ drops of KSOM under oil and cultured in a humidified atmosphere of $5 \% \mathrm{CO}_{2}$ at $37{ }^{\circ} \mathrm{C}$. Fertilization was assessed by the presence of two PN 6-8 h post-insemination and confirmed by the development of 2-cell stage embryos the next day. For these studies day 1 was consider the day of fertilization. Evaluation of embryonic development (2-cell, 4-cell, 8-cell, compacted morula and blastocyst) was done on day 2 , day 3 , day 4 and day 5 .

\section{Mating and embryo culture}

As a control for these experiments, PN-stage embryos were collected from pregnant females and cultured as above. After hCG injection, 2 females were placed overnight with one male. The presence of a vaginal plug accessed the next morning was considered a successful mating. If so, males were separated and females were sacrificed $21 \mathrm{~h}$ post-hCG for collection of PN-stage embryos from the oviduct. 10 to 15 PN-stage embryos were placed in culture as described above.

\section{Processing of embryos for fluorescence microscopy}

In addition to evaluation of embryonic development, control, IVO, IVM and IVM-Rosco-derived embryos were fixed at each day of development, and processed for constitutive (pericentrin) or regulative ( $\gamma$-tubulin) MTOC markers, microtubules, chromatin and lamin B distribution as previously described [14]. Labeling pairs in sequence were (a) mouse monoclonal anti- $\gamma$-tubulin (1:100 dilution, Sigma) followed by Alexa-fluor 568 goat anti-mouse IgG (1:800 dilution, Molecular Probes). This sample was subsequently stained with rabbit polyclonal nuclear lamin B (1:200 dilution) [21, 23] followed by Alexa-fluor 488 goat anti-rabbit IgG (1:600 dilution, Molecular Probes); (b) polyclonal anti-pericentrin (1:200 dilution, Covance, CA) followed by Alexa-fluor 568 goat anti-rabbit IgG (1:800, Molecular Probes). This sample was subsequently stained with rat monoclonal to $\alpha$-tubulin YOL-34 (1:200 dilution) [24] followed by Alexa-fluor 488 goat anti-rat IgG (1:600 dilution, Molecular Probes). Embryos were mounted in a non-compressed way using a mixture of vasolin:lanolin:parafin $(1: 1: 1)$ that was placed on the edges of the coverslip. A $1.5 \mu \mathrm{l}$ of a $50 \%$ glycerol/PBS containing sodium azide and Hoechst $33258(1 \mu \mathrm{g} / \mathrm{ml}$ Polysciences Inc., Warrington, PA) was used as mounting medium. Total cell counts at the blastocyst stage were determined based on the Hoechst 33258 staining of the samples produced. Labeled embryos were analyzed using a Zeiss IM-35 inverted 
microscope and a 50-W mercury arc lamp using 40x and 63x Neofluor objectives. Digital images were collected with a Hamamatsu Orca ER digital camera (model \#C4742-95) interfaced with a Meta Morph Imaging System. A triple band pass dichroic and automated excitation filter selection permitted collection of in-frame images with minimal magnification or spatial distortion.

\section{Confocal microscopy}

Triple labeled samples prepared as described above were analyzed by confocal microscopy using a Zeiss LSM Pascal system mounted on a Zeiss Axioscope II [63x plan Neofluor oil 1.4 numerical aperture (n.a.) or 40x water immersion objectives 1.2 n.a.]. LSM 5 Image Browser was used to analyze samples with complete Z-axis data sets of 30-50 sections per embryo at $0.5-0.7 \mu \mathrm{m}$ depending on the embryonic stage. HeNe laser excitation for each probe permitted complete spatial restoration of Z-series or computed 3-D projections.

\section{Results}

Cytoskeletal organization of IVO and IVM oocytes has been extensively analyzed in terms of their distinct spindle shape, size and MTOCs organization [14]. We have also shown that M-II IVM-Rosco oocytes displayed characteristics closer to their IVO counterparts reflecting pointed shaped spindles and increased number of cytoplasmic MTOCs [15]. Considering that these differences could be used as potential biomarkers of oocyte developmental potential, the present study aimed to carefully assess the pre-implantation development characteristics of embryo-derived in vitro matured oocytes.

In vitro maturation impairs fertilization capacity

IVM and IVM-Rosco oocytes were cultured for $15 \mathrm{~h}$ and the efficiency of maturation was evaluated. As shown in Table 1, both protocols of in vitro maturation were not detrimental in terms of meiotic progression since high percentage of oocytes reached the M-II stage ( $82 \%$ of IVM versus $78 \%$ IVMRosco). Only M-II oocytes were used for IVF. Fertilization

Table 1 Maturation efficiency of IVM and IVM-Rosco oocytes based in two experiments. $\mathrm{N}$ represents total number of oocytes used

\begin{tabular}{lllll}
\hline & GV (\%) & M-I (\%) & M-II (\%) & Degenerated (\%) \\
\hline IVM $(N=100)$ & 3.7 & 6.5 & 82.4 & 7.4 \\
IVM-Rosco $(N=107)$ & 1.8 & 11 & 78 & 9.2 \\
\hline
\end{tabular}

efficiency was assessed by the presence of two visible PN, 6 to $8 \mathrm{~h}$ post-fertilization. IVO, IVM and IVM-Rosco oocytes exhibit distinct IVF efficiencies as shown in Fig. 1. Only $48 \%$ of PN-stage embryos were produced after IVF from IVM oocytes, and $41 \%$ from IVM-Rosco oocytes, in contrast to the $81 \%$ of IVO oocytes that resumed meiosis and formed PN.

In vitro maturation affects developmental potential

We next determined the pre-implantation development of the embryos produced by assessing daily their cleavage rates. Results are shown in Fig. 2.

The cleavage rate of IVM-derived embryos is distinct when compared to IVO-derived embryos: while IVOderived embryos exhibit efficient and synchronous progression until the blastocyst stage, IVM-derived embryos exhibit a variable degree of delay in cleavage rate evident by the simultaneous presence of embryos at different stages of development, as shown in Fig. 2. Therefore, at day 4 while most of the embryos in the IVO group display the compacted morula stage ( $87 \%$ ), IVM-derived embryos exhibit a broader range of cleavage stages, namely 2-cell (29\%), 3-cell (5.6\%), 4-cell (8.3\%), 5-cell (4.1\%), 6-cell (4.1\%), 8-16-cell (7\%) and compacted morula (28\%). Interestingly, when compared to IVM-derived embryos, IVM-Rosco embryos do not display such a broad variability on embryonic stages throughout pre-implantation development suggesting a less delayed progression (on day 4: $35.4 \%$ at 2 -cell, $7.6 \%$ at 3 -cell, and $53.2 \%$ compacted morula).

When comparing pre-implantation development of IVO and IVM-derived embryos at day 5 a dramatic decrease in the percentage of blastocysts produced is observed on both IVM and IVM-Rosco-derived embryos ( $84 \%$ in IVO versus $23 \%$ in IVM and $27 \%$ in IVM-Rosco).

\section{Fertilization Rate (\%) Day 1: PN Check}

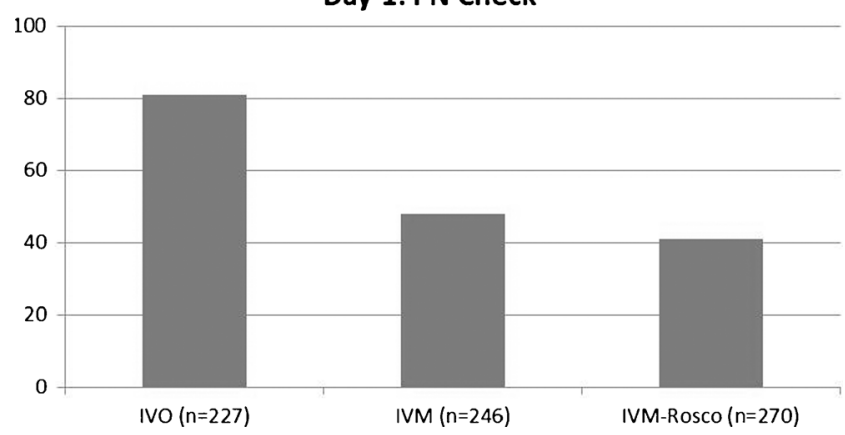

Fig. 1 Fertilization efficiency checked by the presence of a visible PN 6$8 \mathrm{~h}$ post-fertilization of IVO, IVM and IVM-Rosco oocytes ( $81 \%$ for IVO, $48 \%$ for IVM, and $41 \%$ for IVM-Rosco). $\mathrm{N}$ represents total number of embryos used 

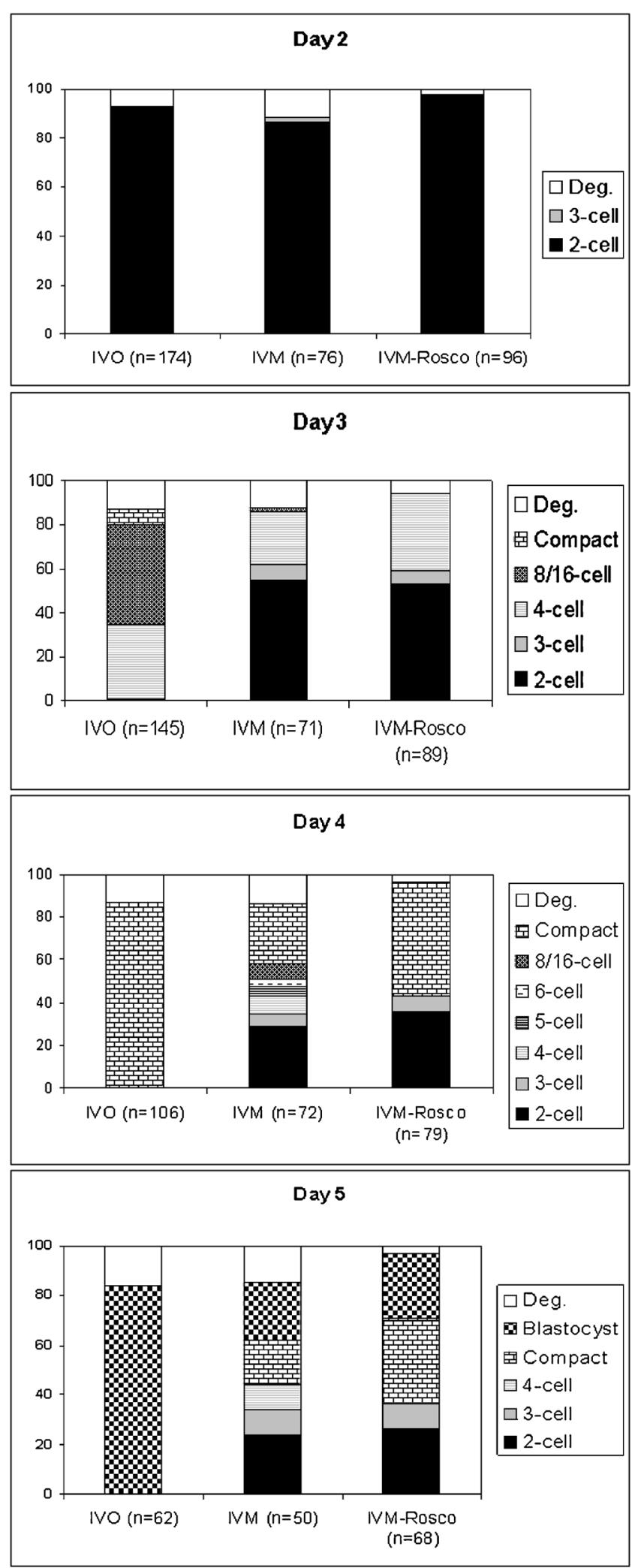

Interestingly, as in the previous day, IVM-Rosco embryos seem to be more advanced than their IVM counterparts, presenting $40 \%$ of compacted morula stage instead of only $20 \%$.
Fig. 2 Development until the blastocyst stage of IVO, IVM and IVMRosco fertilized eggs. Note the synchronism of IVO oocytes contrasting with the asynchronous cleavages of IVM oocytes that exhibit even number of blastomeres on day 4 and day 5 . The efficiency of blastocyst formation is higher in IVO embryos (84 \%) when compared with IVM embryos (23\%). IVM-Rosco embryos exhibit an intermediate synchronism of cleavage rates when compared to both IVO and IVM embryos. The absence of even numbers of blastomeres in the IVM-Rosco group could be an indicator of proper cleavage rates (in contrast to what happen with IVM embryos). Also note the presence of 2-cell stage embryos throughout development in both IVM and IVM-Rosco oocytes

In vitro maturation affects blastomere cleavage symmetry and nuclear morphology

When embryonic morphology was analyzed, several features were observed to differ when IVO, IVM and IVM-Roscoderived embryos were compared. As shown in Table 2, when 2-cell stage embryos were analyzed control-mating (IVO-N) and IVO derived embryos exhibited a symmetric first cleavage plan (94.4 and $71.4 \%$, respectively). However, IVMderived 2-cell stage embryos exhibited increased asymmetries ( $84.8 \%$, Table 2, Fig. 3A-D) when compared to the same stage embryos of IVO-N, IVO and IVM-Rosco groups. Interestingly, IVM-Rosco derived 2-cell stage embryos displayed a more symmetric pattern of first cleavage $(61.5 \%$ symmetric versus $38.5 \%$ asymmetric) that more closely resembled what was observed in IVO 2-cell stage embryos.

Another feature we analyzed was the number of nucleolarprecursor bodies (NPBs) present at the 2-cell stage embryo. IVM-derived 2 cell stage embryos exhibited only 5-6 NPBs in each blastomere ( $n=18$ embryos) contrasting with the 16-17 NPBs present in the IVO group ( $n=18$ embryos). Interestingly, IVM-Rosco 2-cell stage embryos exhibited an intermediate number of NPBs in each blastomere (9-10, $n=10$ embryos). Representative examples of this feature are shown in Fig. 3.

In vitro maturation affects cell number at the blastocyst stage

Total number of cells at the blastocyst stage was evaluated at day 5 based on the Hoechst 33258 nuclei staining. As shown in Fig. 4, IVO-derived blastocysts exhibited comparable cell numbers with the blastocysts produced from control-mating and analyzed at day $5(73.35 \pm 12,43$ in IVO versus $72.3 \pm 9.26$ control-mating). In contrast, IVM-derived blastocysts display a low number of cells (56 \pm 7.22$)$. Interestingly, IVM-Rosco-

Table 2 Cleavage plan symmetries in 2-cell stage embryos

\begin{tabular}{lll}
\hline & Symmetric (\%) & Assymetric (\%) \\
\hline Control-mating $(n=18)$ & 94.4 & 5.6 \\
IVO $(n=28)$ & 71.4 & 28.6 \\
IVM $(n=33)$ & 15.2 & 84.8 \\
IVM-ROSCO $(n=26)$ & 61.5 & 38.5 \\
\hline
\end{tabular}




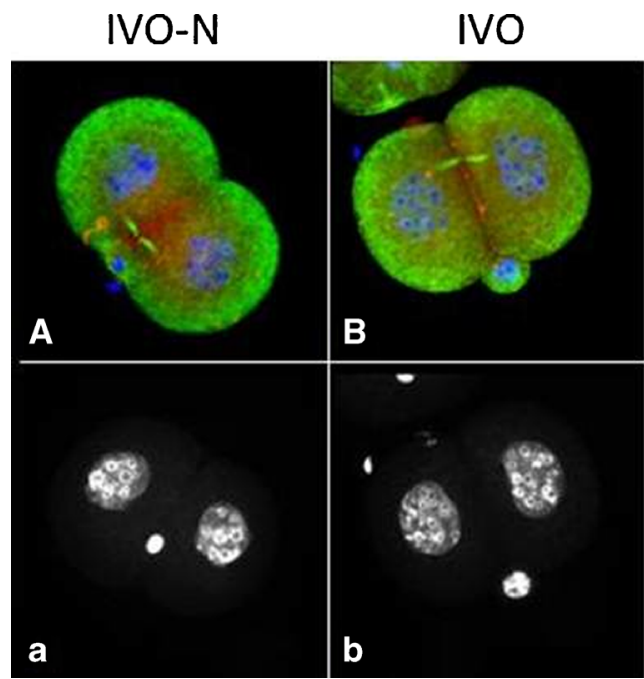

Fig. 3 2-cell stage IVO-N, IVO, IVM and IVM-Rosco embryos labeled for tubulin (green), pericentrin (red) and chromatin (blue) (A-D), corresponding chromatin staining (a-d) depicting the number of small nucleoli on each blastomere. Note the asymmetric size of the two blastomeres of

derived blastocysts exhibit an intermediate number of blastomeres between IVO and IVM-embryos (63.4 \pm 9.82$)$.

Centrosomal-microtubule organization of cleavage and blastocyst stage embryos

Since IVO, IVM and IVM-Rosco M-II oocytes were previously shown to exhibit distinct cytoskeleton organization [14, 15], we next examined distribution of the centrosomalmicrotubule complex among the embryos produced. However, although the oocytes exhibited distinct cytoskeleton organization, these differences were not evident in the embryos produced. When microtubule, nuclear lamina organization and centrosomal distribution were evaluated no differences were observed between IVO, IVM and IVM-Rosco derived embryos. However, when pericentrin and $\gamma$-tubulin

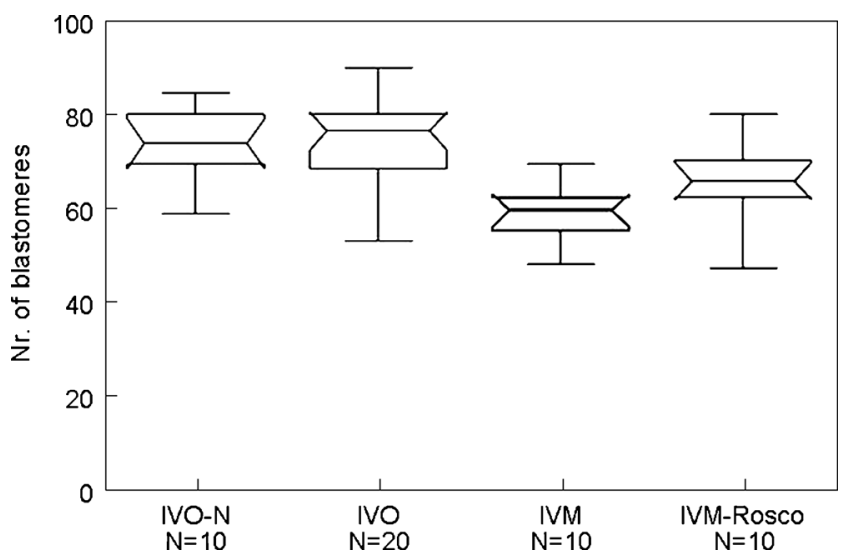

Fig. 4 Total cell counts of IVO-N (control), IVO, IVM and IVM-Rosco blastocysts. Note the significantly less number of cells on the IVM blastocysts compared with both IVO-N and IVO. IVM-Rosco blastocysts exhibit an intermediate phenotype between IVM and IVO embryos

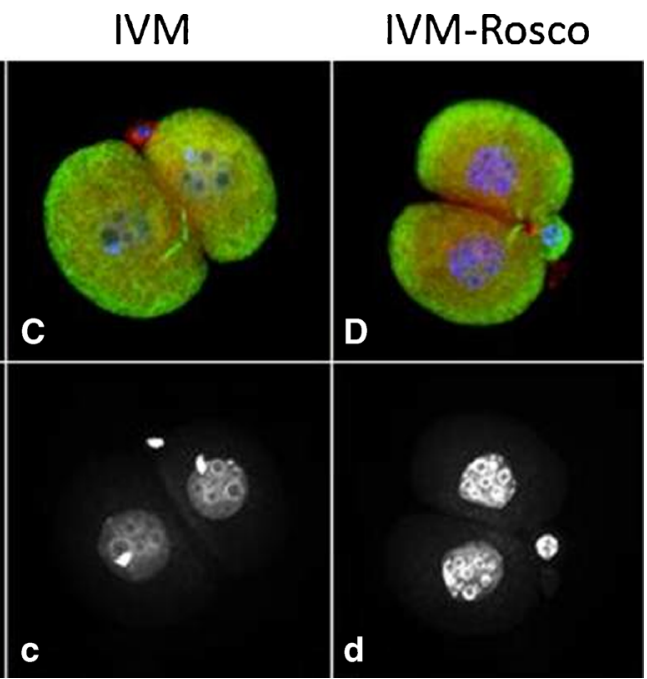

IVM 2-cell stage embryos (C) when compared to IVO (B) and IVMRosco (D). Also note that IVM 2-cell stage embryos (c) exhibit fewer nucleoli when compared to both IVO (b) and IVM-Rosco embryos (d), probably denoting distinct transcriptional activity

distribution was evaluated, a distinct organization of these centrosomal markers was observed in all groups as shown in Fig. 5.

Pericentrin exhibits a dispersed cytoplasmic organization during the first cleavage stages until compaction (occurring between 8 and 16-cell stage embryo) where it starts showing a dispersed but apical distribution (Fig. 5c). In contrast, although $\gamma$-tubulin also exhibits a dispersed cytoplasmic distribution during the first cleavage stages, it starts showing punctuate and perinuclear staining in compacted stage embryos (Fig. 5C). Interestingly, whenever a spindle is observed $\gamma$ tubulin is distributed throughout the spindle (Fig. 5A, B). In contrast, pericentrin is only observed at the spindle poles (Fig. 5a,b). This differential distribution of $\gamma$-tubulin and pericentrin was noted until the blastocyst stage with $\gamma$ tubulin distributed throughout the spindle and pericentrin restricted to the spindle poles (Fig. 5D and d, respectively). Regardless of the embryonic stage, blastomeres exhibit pointed-shape spindles that assume a very focused appearance at the blastocyst stage as shown in Fig. 6.

Nuclear lamina distribution, as expected, always exhibited a regular pattern outlining the nucleus of each blastomere or, whenever a spindle was observed, dispersed through the cytoplasm of each dividing blastomere (Fig. 7).

\section{Discussion}

In the present study, the developmental competence of IVM and IVM-Rosco oocytes was directly compared with IVO oocytes. The hypothesis that the distinct cytoskeleton configurations found in IVO, IVM and IVM-Rosco oocytes reflects 


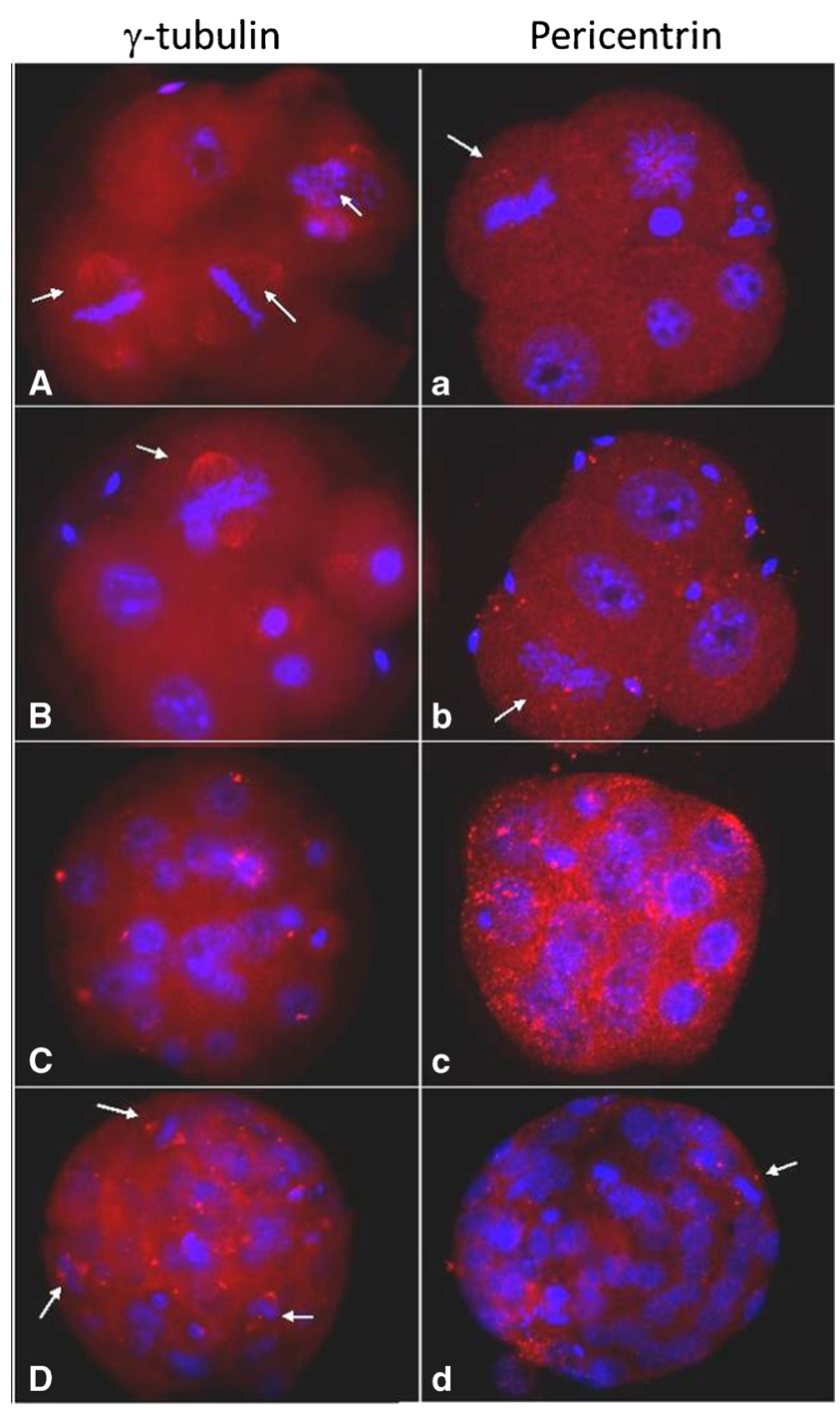

Fig. $5 \gamma$-tubulin (A-D) and pericentrin (a-d) pattern in mouse embryos. Chromatin is shown in blue (Hoechst staining). Note distribution of $\gamma$ tubulin (A, B), not pericentrin $(\mathbf{a}, \mathbf{b})$, forming the spindle during the first cleavages. $\gamma$-tubulin exhibits a punctuate perinuclear localization at compacted morula $(\mathbf{C})$ and forms the spindle proper in blastocysts stage embryos (D). Pericentrin exhibits an apical distribution in 8-cell stage embryos (c) and forms the spindle poles in the blastocyst stage (d). Arrow indicate cell in metaphase

distinct pre-implantation developmental competence was addressed [14-16]. To deal with this question we have performed IVF and assessed subsequent pre-implantation development. The embryos produced were daily observed, cleavage rates assessed, blastomere size evaluated and the total number of cells counted at the blastocyst stage. Additionally, to characterize cytoskeleton organization of the embryos produced, embryos were fixed and stained for evaluation of microtubule patterning, regulative ( $\gamma$-tubulin) and constitutive (pericentrin) centrosomal markers, and nuclear lamina distribution. Our results show that IVM oocytes, as predicted, impaired fertilization efficiency, compromise developmental competence, and reveal abnormalities in blastomere symmetry and in nuclear morphology throughout cleavage and until the blastocyst stage.

Interestingly, IVM-Rosco-derived embryos show less dramatic perturbations, bringing it closer to the IVO-derived embryo characteristics, suggesting that the unique properties that underlie coordination of nuclear and cytoplasmic events during oocyte maturation may be preserved with the use of a cell cycle arresting agent during oocyte maturation.

Both groups of IVM-derived embryos exhibit low fertilization efficiencies

We have previously shown that M-II IVM oocytes exhibit loosen barrel-shaped spindles contrasting with the compacted pointed-shape spindles, cortically localized, of IVO oocytes [14, 15, 25]. Additionally, when compared to IVO spindles, IVM spindles exhibited decreased phosphorylation levels detected by the mitotic phosphoprotein antibody MPM-2 [14]. Since important cell cycle factors involved in egg activation and pronuclear formation were found to be localized to the spindle, especially to the spindle poles [26-28] a miss-localization of these factors could have deleterious implications in egg activation and fertilization efficiency. Furthermore, depletion of MTOC material from the cytoplasm was found to occur in IVM oocytes $[14,15]$, and this depletion predicted to increase with second polar body emission at fertilization [16]. Since maternal $\gamma$-tubulin has been shown to be critical for microtubule reorganization during fertilization and pronuclear apposition [29], it was expected that IVM oocytes, which are depleted from $\gamma$-tubulin stores in their cytoplasm, would exhibit impaired fertilization efficiency. Although IVMRosco M-II oocytes have been shown to exhibit increased number of cytoplasmic MTOCs, and cortically localized pointed-shape spindles [15] their fertilization efficiency was also low. These results may imply that the described configurations have a longer term-effect seen only when analyzing pre-implantation development. However, it could also be linked to a negative effect of the drug on the ability of the oocyte to be fertilized, as it has been previously described in porcine oocytes [30]. However, further studies are needed to determine the exact impact of this drug on the developmental competence of matured mouse oocytes.

IVM directly impacts pre-implantation development

The dynamics of the preimplantation embryo cell cycle, specifically the timing and pace of the first embryonic divisions has been correlated with the developmental potential of human embryos [31-33]. Since the number of cytoplasmic MTOCs in the oocyte was considered an important maternal factor necessary to support first embryonic cleavages [16, 
Fig. 6 IVO (A-C) and IVM $(\mathbf{a}-\mathbf{c})$ embryos stained for $\alpha$-tubulin evidencing pointed shape spindles. 4 cell-embryo $(\mathbf{A}, \mathbf{a})$, compacted morula $(\mathbf{B}, \mathbf{b})$ and blastocyst $(\mathbf{C , c})$

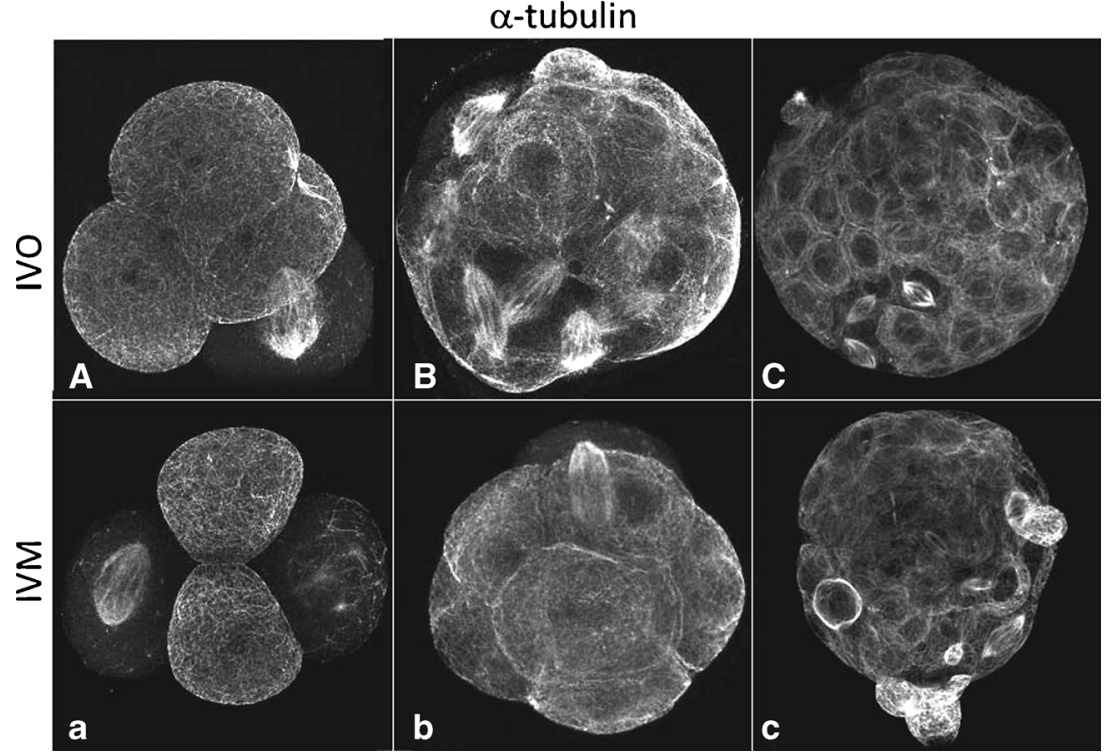

34-36] and given that IVO, IVM and IVM-Rosco oocytes displayed characteristically distinct number of cytoplasmic MTOCs [14, 15], we further analyzed cleavage rates of the embryos produced.

When cleavage rates were compared between these groups, IVO oocytes exhibited synchronous and efficient developmental progression until the blastocyst stage while IVM and IVM-Rosco oocytes displayed a delayed progression until the blastocyst stage. This delay was clearer in IVM than in IVMRosco embryos, both at day 4 and 5 depicting deficient cell cycle progression. IVM-Rosco embryos exhibited a more effective cell cycle progression probably due to a spatial organization of their microtubule-centrosomal complex closer to the IVO situation.
In addition, IVM-Rosco blastocysts exhibited higher number of cells at the blastocyst stage when compared to IVM oocytes. If the maternal MTOC legacy is an important factor to support cell cycle progression during preimplantation development [35, 37], it would be expected that IVM-Rosco oocytes once fertilized exhibited increased cell cycle progression during preimplantation development when compared to their IVM counterparts. Indeed, not only is the total cell number in the blastocyst stage higher, but also developmental progression until the blastocyst stage seems more efficient in IVM-Rosco embryos than in IVM embryos.

One of the major events during preimplantation development is the maternal to zygotic transition that, in the mouse, occurs at the 2-cell stage when important embryonic genes are
Fig. 7 2-cell embryo (a), 8-cell embryo (b), compacted morula (c) and blastocyst (d) depicting nuclear lamina (lamin B, green), $\gamma$-tubulin (red) and chromatin (Hoechst 33258, blue). Note that nuclear lamina is dispersed in the cytoplasm in dividing cells (arrows)

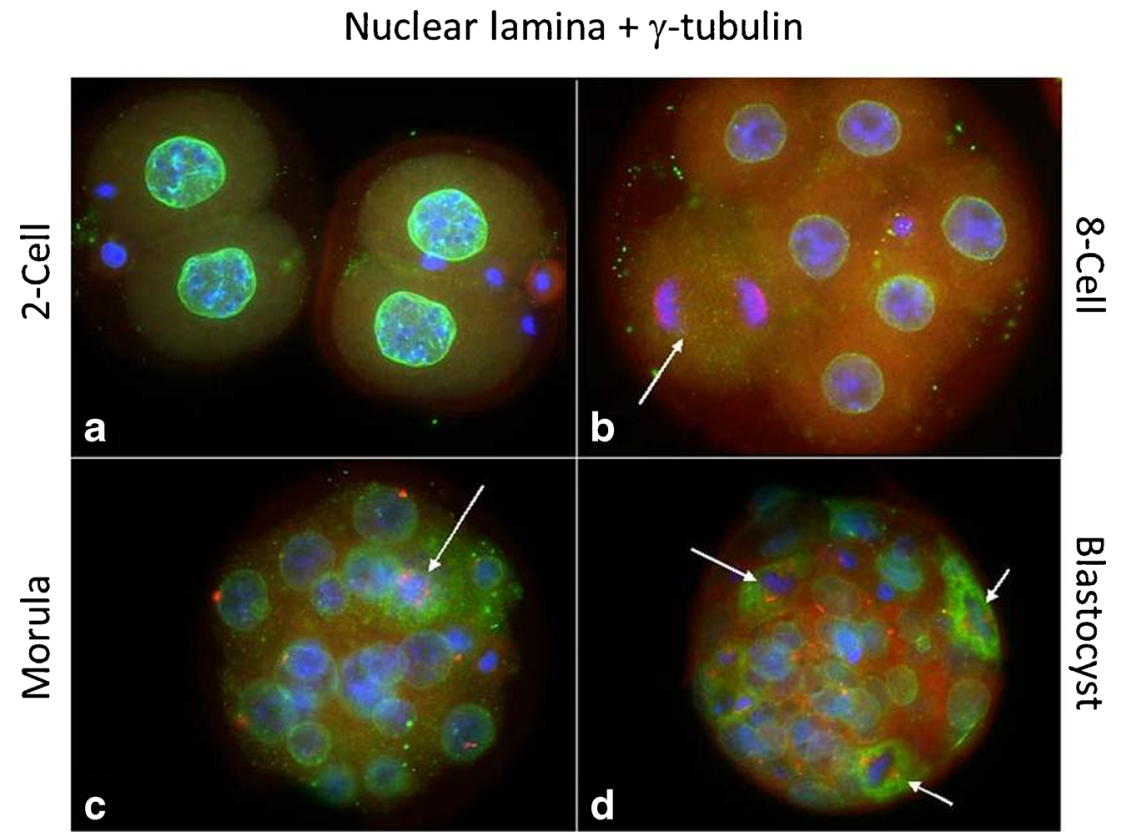


switched on [38]. When 2-cell stage embryos were analyzed, IVM embryos exhibited distinct numbers of nucleoli precursor bodies (NPBs) when compared to IVO embryos. NPBs were found to contain the major proteins for rRNA synthesis $[39,40]$. Since IVM embryos exhibit less NPBs than IVO or IVM-Rosco 2-cell stage embryos, and although further studies are needed to prove this hypothesis, we speculate that abnormalities exist in genome activation and ultimately nuclear function in IVM embryos.

Actin cytoskeleton has been implicated in cell polarity, chromosome migration and asymmetric divisions during oocyte maturation [41-43]. An intact actin network has also been responsible for the symmetric cell divisions of the mouse oocyte [44]. IVM-derived 2-cell embryos exhibited asymmetrical first cleavages denoting a loss of spatial control of cytokinesis after PN apposition. The same did not happen with IVO and IVM-Rosco 2-cell stage embryos where the appearance of a symmetric first cleavage plan was observed. A deficiency in the first cleavage plan site that occurs upon fertilization originates asymmetric blastomeres and may compromise development to the blastocyst stage, as is well known in the human $[44,45]$. Unequal distribution of important cell cycle regulators after the first cleavage might affect subsequent development of the embryo [46].

Despite the involvement of the cortical microfilaments, what exactly defines the place where the first cleavage plane forms remains a controversial topic [45, 47]. However, it is interesting to correlate the distinct spatial organization of the centrosome-microtubule complex in IVM M-II oocytes to a loss of spatial polarity during M-II arrest [16]. Our results suggest that delaying the onset of cell cycle progression during oocyte maturation has a benefit effect in terms of cortex remodeling culminating in symmetric cleavage upon fertilization of IVM-Rosco-derived embryos.

Collectively, the present study showed that fertilization efficiency, pre-implantation developmental progression, blastomere division symmetries, blastomere nuclear morphology and blastomere cell number at the blastocyst stage are impacted in embryos produced from IVM oocytes. Manipulations during the early stages of IVM aimed to arrest cell cycle progression were partially effective in correcting these perturbations highlighting the potential utility of cell cycle synchronizing agents like roscovitine. Given the growing emphasis on time-lapse microscopy as a prognostic tool for embryo quality evaluation, the present study identifies novel embryonic cytoskeletal biomarkers that take their origin from conditions used during in vitro oocyte maturation. We suggest that these distinct cytoskeleton characteristics may be considered as additional biomarkers for mouse oocyte developmental quality. Further studies are needed in tractable experimental rodent and non-human primate and human oocytes to assess the value of using cell cycle arresting drugs during IVM protocols. If benefits upon developmental competence of IVM oocytes are confirmed, this will surely have a strong impact upon current human ART IVM procedures and outcomes.

Acknowledgments We would like to thank to Rudi Rottenfusser (Zeiss) and Louis Kerr of the Central Microcopy Facility of the Marine Biological Laboratory, Woods Hole for their provision of imaging resources used in this work. We also recognize the ESHE Fund and the Hall Family Foundation for continued support of this research.

Grant support This work was supported by Fundação para a Ciência e a Tecnologia \#SFRH/BD/2757/2000 (AS), POCTI/ESP/43628/2000 (CEP), March of Dimes Birth Defects Foundation 01-248 (DFA), NIH HD20068 (DFA)

Open Access This article is distributed under the terms of the Creative Commons Attribution License which permits any use, distribution, and reproduction in any medium, provided the original author(s) and the source are credited.

\section{References}

1. Li R, Albertini DF. The road to maturation: somatic cell interaction and self-organization of the mammalian oocyte. Nat Rev Mol Cell Biol. 2013;14(3):141-52.

2. Jones KT, Lane SIR, Holt JE. Start and stop signals of oocyte meiotic maturation. In: Coticchio G, Albertini DF, De Santis L, editors. Oogenesis. New York: Springer; 2013.

3. Eppig JJ. Coordination of nuclear and cytoplasmic oocyte maturation in eutherian mammals. Reprod Fertil Dev. 1996;8:485-9.

4. Downs SM. Regulation of the G2/M transition in rodent oocytes. Mol Reprod Dev. 2010;77:566-85.

5. Edwards RG. Maturation in vitro of mouse, sheep, cow, pig, rhesus monkey and human ovarian oocytes. Nature. 1965;208:349-51.

6. Pincus G, Enzmann EV. The comparative behavior of mammalian eggs in vivo and in vitro. J Exp Med. 1935;62:665-75.

7. Hardy K, Wright CS, Franks S, Winston RML. In vitro maturation of oocytes. Br Med Bull. 2000;56:588-602.

8. Combelles CMH, Albertini DF. Assessment of oocyte quality following repeated gonadotropin stimulation in the mouse. Biol Reprod. 2003;2003:812-21.

9. Nishi Y, Takeshita T, Sato K, Araki T. Change of the mitochondrial distribution in mouse ooplasm during in vitro maturation. J Nippon Med Sch. 2003;70:408-15.

10. De Matos DG, Nogueira D, Cortvrindt R, Herrera C, Adriaenssens T, Pasqualini RS, et al. Capacity of adult and prepubertal mouse oocytes to undergo embryo development in the presence of cysteamine. Mol Reprod Dev. 2003;64:214-8.

11. Eichenlaub-Ritter U, Peschke M. Expression in in vivo and in vitro growing and maturing oocytes: focus on regulation of expression at the translational level. Hum Reprod Update. 2002;8:21-41.

12. Rodriguez K, Farin CE. Gene transcription and regulation of oocyte maturation. Reprod Fertil Dev. 2004;16:55-67.

13. Lucifero D, Mertineit C, Clarke HJ, Bestor TH, Trasler JM. Methylation dynamics of imprinted genes in mouse germ cells. Genomics. 2002;79:530-8.

14. Sanfins A, Lee G, Plancha C, Overstrom E, Albertini DF. Distinctions in meiotic spindle structure and assembly during in vitro and in vivo maturation of mouse oocytes. Biol Reprod. 2003;69:2059-67.

15. Sanfins A, Plancha C, Overstrom E, Albertini D. Meiotic spindle morphogenesis in in vivo and in vitro matured mouse oocytes: 
insights into the relationship between nuclear and cytoplasmic quality. Hum Reprod. 2004;19:2889-99.

16. Plancha C, Sanfins A, Rodrigues P, Albertini D. Cell polarity during folliculogenesis and oogenesis. RBM Online. 2005;10(4):478-84.

17. Barrett SL, Albertini DF. Allocation of gamma-tubulin between oocyte cortex and meiotic spindle influences asymmetric cytokinesis in the mouse oocyte. Biol Reprod. 2007;76(6):949-57.

18. Barrett SL, Albertini DF. Cumulus cell contact during oocyte maturation in mice regulates meiotic spindle positioning and enhances developmental competence. J Assist Reprod Genet. 2010;27:29-39.

19. Gilchrist RB. Recent insights into oocyte-follicle cell interactions provide opportunities for the development of new approaches to in vitro maturation. Reprod Fertil Dev. 2010;23:23-31.

20. Sanchez F, Smitz J. Molecular control of oogenesis. Biochim Biophys Acta. 2012;12:1896-912. Dec 1822.

21. Erbach G, Lawitts J, Papaioannou V, Biggers JD. Differential growth of the mouse preimplantation embryo in chemically defined media. Biol Reprod. 1994;50:1027-33.

22. Summers C, McGinnis L, Lawitts J, Raffin M, Biggers J. IVM of mouse ova in a simplex optimized medium supplemented with amino acids. Hum Reprod. 2000;15:1791-801.

23. Gerace L, Blum A, Blobel G. Immunocytochemical localization of the major polypeptides of the nuclear pore complex-lamina fraction. Interphase and mitotic distribution. J Cell Biol. 1978;79:546-66.

24. Kilmartin J, Wright B, Milstein C. Rat monoclonal antitubulin antibodies derived by using a new nonsecreting rat cell line. J Cell Biol. 1982;93:576-82.

25. Ibanez E, Sanfins A, Combelles CMH, Overstrom EW, Albertini DF. Strains-dependent meiotic competence properties of mouse oocytes. Reproduction. 2005;130:845-55.

26. Verlhac M-H, De Pennart H, Maro B, Cobb MH, Clarke HJ. MAP kinase becomes stably activated at metaphase and is associated with microtubule-organizing centers during meiotic maturation of mouse oocytes. Dev Biol. 1993;158:330-40.

27. Pahlavan G, Polanski Z, Kalab P, Golsteyn R, Nigg E, Maro B. Characterization of polo-like kinase 1 during meiotic maturation of the mouse oocyte. Dev Biol. 2000;220:392-400.

28. Fan H, Huo L, Meng X, Zhong Z, Hou Y, Chen D, et al. Involvement of calcium/calmodulin-dependent protein kinase II (CaMKII) in meiotic maturation and activation of pig oocytes. Biol Reprod. 2003;69: $1552-64$.

29. Shin M, Kim N. Maternal gamma-tubulin is involved in microtubule reorganization during bovine fertilization and parthenogenesis. Mol Reprod Dev. 2003;64:438-45.

30. Marchal R, Tomanek M, Terqui M, Mermillod P. Effects of cell cycle dependent kinases inhibitor on nuclear and cytoplasmic maturation of porcine oocytes. Mol Reprod Dev. 2001;60:65-73.

31. Adjuk A, Zernicka-Goetz, M. Advances in embryo selection methods. F1000 Biology Reports 2012; 4:11.
32. Wong CC, Loewke KE, Bossert NL, Behr B, De Jonge CJ, Baer TM, et al. Non-invasive imaging of human embryos before embryonic genome activation predicts development to the blastocyst stage. Nat Biotechnol. 2010;28:1115-11121.

33. Lundin K, Bergh C, Hardarson T. Early embryo cleavage is a strong indicator of embryo quality in human IVF. Hum Reprod. 2001;16: 2652-7.

34. Courtois A, Schuh M, Ellenberg J, Hiiragi T. The transition from meiotic to mitotic spindle assembly is gradual during early mammalian development. J Cell Biol. 2012;198:357.

35. Maro B, Howlett SK, Webb M. Non-spindle microtubule organizing centers in metaphase II - arrested mouse oocytes. J Cell Biol. 1985;101:1665-72.

36. Gueth-Hallonet C, Antony C, Aghion J, Santa-Maria A, LajoieMazenc I, Wright B, et al. Gamma-tubulin is present in acentriolar MTOCs during early mouse development. J Cell Sci. 1993;105: 157-66.

37. Maro B, Kubiak J, Gueth C, De Pennart H, Houliston E, Weber M, et al. Cytoskeleton organization during oogenesis, fertilization and perimplantation development of the mouse. Int J Dev Biol. 1990;34: $127-37$.

38. Telford N, Watson A, Schultz G. Transition from maternal to embryonic control in early mammalian development: a comparison of several species. Mol Reprod Dev. 1990;26:90-100.

39. Ferreira J, Carmo-Fonseca M. The biogenesis of the coiled body during early mouse development. Development. 1995;121:601-12.

40. Zatsepina O, Bouniol-Baly C, Amirand C. Functional and molecular reorganization of the nucleolar apparatus in maturing mouse oocytes. Dev Biol. 2000;223:354-70.

41. Yi K, Unruh JR, Deng M, Slaughter BD, Rubinstein B, Li R. Dynamic maintenance of asymmetric meiotic spindle position through Arp2/3 complex-driven cytoplasmic streaming in mouse oocytes. Nat Cell Biol. 2011;13:1252-8.

42. Wolke U, Jezuit EA, Priess JR. Actin-dependent cytoplasmic streaming in C. elegans oogenesis. Development. 2007;134:2227-36.

43. Na J, Zernicka-Goetz M. Asymmetric positioning and organization of the meiotic spindle of mouse oocytes requires CDC42 function. Curr Biol. 2006;16(12):1249-54.

44. Chew TG, Lorthongpanich C, Ang WX, Knowles BB, Solter D. Symmetric cell division of the mouse zygote requires an actin network. Cytoskeleton. 2012;69:1040-6.

45. Zernicka-Goetz M. First cell fate decisions and spatial patterning in the early mouse embryo. Semin Cell Dev Biol. 2004;15:563-72.

46. Cooke S, Tyler J, Driscoll GL. Meiotic spindle location and identification and its effect on embryonic cleavage plane and early development. Hum Reprod. 2003;18:2397-405.

47. Hiiragi T, Solter D. First cleavage plane of the mouse egg is not predetermined but defined by the topology of the two apposing pronuclei. Nature. 2004;430:360-4. 